\title{
Boolean Zero Square (BZS) Semigroups
}

\author{
G.A. Pinto \\ Department of Mathematics, College of Science, Sultan Qaboos University, P.O. Box 36, \\ Al-Khoud, PC 123, Muscat, Sultanate of Oman. Email: goncalo@squ.edu.om
}

ABSTRACT: We introduce a new class of semigroups, that we call BZS - Boolean Zero Square-semigroups. A semigroup $S$ with a zero element, 0 , is said to be a BZS semigroup if, for every $x \in S$, we have $x^{2}=x$ or $x^{2}=0$. We obtain some properties that describe the behaviour of the Green's equivalence relations $\mathcal{R}, \mathcal{L}, \mathcal{H}$ and $\mathcal{D}$. Necessary and sufficient conditions for a BZS semigroup to be a band and an inverse semigroup are obtained. A characterisation of a special type of BZS completely 0-simple semigroup is presented.

Keywords: BZS semigroup; Green's relations; Regular; Completely 0-simple; Inverse and band.

أشباه الزمر البولانية ذات المربع الصفري

ج. أ. بينتو

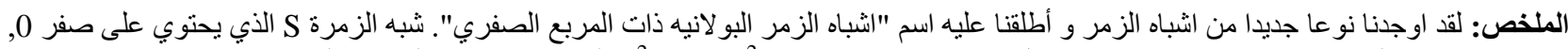

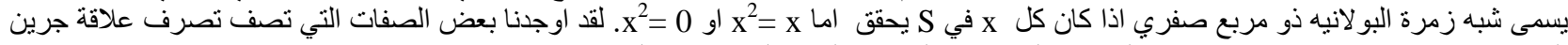

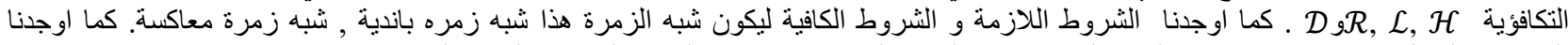

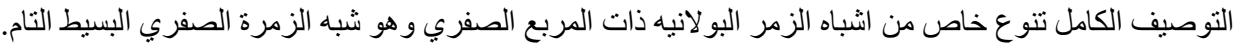
الكلمات المفتاحية: شبه زمرة البو لانيه ذو مربع صفري، علاقة جرين، منتظم، الصفري البسيط التام، معكوس و فرقة. 


\section{G.A. PINTO}

\section{Introduction}

W

e shall use standard semigroup notation, that can be found, for example, in [1]. If $S$ is a semigroup, $E(S)$ represents the set of idempotents of $S$, and $V(x)$ denotes the set of inverses of an element $x$ in $S$. We recall that the natural order $\leq_{n}$ on the idempotents of a regular semigroup is defined by

$$
e \leq_{n} f \Leftrightarrow e=e f=f e
$$

In [2], Farag and Tucci introduced the notion of a Boolean Zero Square (BZS) ring as an associative ring, not necessarily commutative and not necessarily with identity, such that every non-zero element of $R$ is either idempotent or nilpotent of index 2 , that is,

$$
(\forall x \in R) \quad x^{2}=x \quad \vee \quad x^{2}=0
$$

The structure of BZS rings is investigated, in [2] and in [3].

It is possible to find in the literature several papers in Boolean Zero rings, and as a sample we refer to [4] in order to several constructions in this structure.

Here we present a generalisation of these notions to semigroup theory, starting by introducing the following concept, that follows naturally from Ring theory.

Definition. A semigroup $S$, with element zero 0 , is said to be a BZS semigroup if, for every element $x$ in $S$, we have $x^{2}=x$ or $x^{2}=0$.

In the ring case, there are more tools available due to the presence of two operations: addition and multiplication. This fact has, as a consequence, that the majority of the results obtained in [2] and [3] cannot be replicated to the semigroup case.

One of the results obtained in [2] is that in a BZS ring, the set of nilpotent elements, is an ideal of the ring. This property does not hold in a general BZS semigroup, as it can easily be seen in Example 2 below. This happens because the result only mentions the multiplicative operation, although its proof uses the additive operation heavily. We obtain in Theorem 8 that in a BZS commutative semigroup the set of its nilpotent elements, is an ideal of the semigroup.

In what follows, unless otherwise stated, $S$ will always denote a BZS semigroup. We denote

$$
E=\left\{x \in S \mid x^{2}=x\right\} \quad \text { and } N=\left\{x \in S \mid x^{2}=0\right\}
$$

respectively, the set of idempotent elements and the set of nilpotent elements of $S$.

Let us present some basic properties that hold in any such semigroup $S$.

$(\forall x \in S) \quad x^{3}=x^{2}$

For any $x \in S$, we have two cases to consider:

i) $x^{2}=x \Rightarrow x \cdot x^{2}=x \cdot x \Rightarrow x^{3}=x^{2}$

ii) $x^{2}=0 \Rightarrow x \cdot x^{2}=x \cdot 0 \Rightarrow x^{3}=0 \Rightarrow x^{3}=x^{2}$

which proves the result.

$(\forall x \in S) \quad x^{4}=x^{2} \quad$ and $\quad x^{2} \in E(S)$

This follows immediately from (1).

$E \cap N=\{0\}$

In fact, if $x \in E \cap N$, we have $x=x^{2}=0$, and the result follows.

$(\forall x, y \in S) \quad x y \in E \backslash\{0\} \quad \Leftrightarrow \quad y x \in E \backslash\{0\}$

Let us, assume that $x y \in E \backslash\{0\}$, that is, $(x y)^{2}=x y$, with $x y \neq 0$. If $y x=0$ then,

$$
\begin{aligned}
y x=0 & \Rightarrow x \cdot y x \cdot y=x \cdot 0 \cdot y \\
& \Rightarrow(x y)^{2}=0
\end{aligned}
$$




\section{BOOLEAN ZERO SQUARE (BZS) SEMIGROUPS}

which is a contradiction, and therefore we can conclude that $y x \neq 0$. Also, if $(y x)^{2}=0$ then, using (1), we have

$$
\begin{aligned}
(y x)^{2}=0 & \Rightarrow x(y x)^{2} y=x \cdot 0 \cdot y \\
& \Rightarrow(x y)^{3}=0 \\
& \Rightarrow(x y)^{2}=0 \\
& \Rightarrow x y=0
\end{aligned}
$$

which is also a contradiction. So, $(y x)^{2}=y x \neq 0$, which means that, $y x \in E \backslash\{0\}$.

Similarly, we prove the reverse implication.

$$
(\forall x, y \in S) \quad x y \in N \Leftrightarrow y x \in N
$$

If $x y \in N$ then, by (3), $x y \notin E \backslash\{0\}$ and therefore by (4), $y x \notin E \backslash\{0\}$, that is $y x \in N$. The converse implication follows similarly.

Now, let us present some examples to illustrate this concept, which show that they can be found in a wide variety of very well-known classes of semigroups, such as bands, completely 0 -simple semigroups and inverse semigroups.

Example 1. Any band, $B$, with zero is clearly, a BZS semigroup, with $E=B$ and $N=\{0\}$.

Example 2. In a context of ordered semigroup theory, Blyth and McFadden presented in [5] a semigroup which has proved to be very helpful in describing several classes of ordered semigroups. More details of the relevance and properties of this semigroup can also be found in [6]. It can be defined by $N_{5}=\{u, e, f, a, b\}$ with the following Cayley table:

\begin{tabular}{c|ccccc} 
& $u$ & $e$ & $f$ & $a$ & $b$ \\
\hline$u$ & $u$ & $u$ & $f$ & $f$ & $b$ \\
$e$ & $e$ & $e$ & $a$ & $a$ & $b$ \\
$f$ & $u$ & $b$ & $f$ & $b$ & $b$ \\
$a$ & $e$ & $b$ & $a$ & $b$ & $b$ \\
$b$ & $b$ & $b$ & $b$ & $b$ & $b$
\end{tabular}

It follows directly from the table that $N_{5}$ is a BZS semigroup, $b$ is its zero element, $N=\{a, b\}$ and $E=\{u, e, f, b\}$. This semigroup appears in a different context, as an example of a completely 0-simple semigroup that it is not orthodox. Routine calculations show that it is 0 -simple

$$
N_{5}=N_{5} u N_{5}=N_{5} e N_{5}=N_{5} f N_{5}=N_{5} a N_{5}
$$

and, for example, $f$ is a primitive idempotent. Since $e f \neq f e$, we can state that $N_{5}$ is not an orthodox semigroup.

Example 3. Consider the completely 0-simple semigroup $S=(I \times G \times \Lambda) \cup\{0\}$ with operation

$$
\begin{aligned}
& (i, a, \lambda)(j, b, \mu)=\left\{\begin{array}{ccc}
\left(i, a p_{\lambda j} b, \mu\right) & \text { if } & p_{\lambda j} \neq 0 \\
0 & \text { if } & p_{\lambda j}=0
\end{array}\right. \\
& (i, a, \lambda) 0=0=0(i, a, \lambda)=00
\end{aligned}
$$

where $G^{0}=G \cup\{0\}$ is a zero group, with $G=\langle x\rangle$ an order two cyclic group, $I, \Lambda$ are non-empty index sets and $P=\left[p_{\lambda i}\right]$ is a $\Lambda \times I$ sandwich matrix with entries in $G^{0}$, and all the non-zero entries of $P$ are equal to $x$. Recall that every row and column of $P$ has at least a non-zero entry.

Consider $T=\{(i, x, \lambda) \in S\} \cup\{0\}$ a subset of $S$, and let $(i, x, \lambda),(j, x, \mu) \in T$. We have the following possibilities for the element $p_{\lambda j}$ :

$$
\begin{aligned}
& \text { If } p_{\lambda j} \neq 0 \text {, then }(i, x, \lambda)(j, x, \mu)=\left(i, x p_{\lambda j} x, \mu\right)=(i, x x x, \mu)=(i, x, \mu) \in T \\
& \text { If } p_{\lambda j}=0 \text {, then }(i, x, \lambda)(j, x, \mu)=0 \in T
\end{aligned}
$$




\section{G.A. PINTO}

and we can say that $T$ is a semigroup, with the induced semigroup operation. Also, for any $(i, x, \lambda) \in T$, we have that

$$
(i, x, \lambda)^{2}=\left\{\begin{array}{ccc}
(i, x, \lambda) & \text { if } & p_{\lambda i} \neq 0 \\
0 & \text { if } & p_{\lambda i}=0
\end{array}\right.
$$

Therefore, $T$ is a BZS semigroup.

Example 4. Consider the following set of $2 \times 2$ real matrices

$$
S=\left\{I, A, E_{11}, E_{12}, E_{21}, E_{22}, O\right\}=\left\{\left[\begin{array}{ll}
1 & 0 \\
0 & 1
\end{array}\right],\left[\begin{array}{ll}
0 & 1 \\
1 & 0
\end{array}\right],\left[\begin{array}{ll}
1 & 0 \\
0 & 0
\end{array}\right],\left[\begin{array}{ll}
0 & 1 \\
0 & 0
\end{array}\right],\left[\begin{array}{ll}
0 & 0 \\
1 & 0
\end{array}\right],\left[\begin{array}{ll}
0 & 0 \\
0 & 1
\end{array}\right],\left[\begin{array}{ll}
0 & 0 \\
0 & 0
\end{array}\right]\right\}
$$

It is well known that $S$ with the usual matrix multiplication is an inverse semigroup (see, for example [7, Section 7.6, Exercise 1]). $S$ is not a BZS semigroup, since $A^{2}=A A=I \neq A, O$.

But if we consider the subset

$$
T=\left\{I, E_{11}, E_{12}, E_{21}, E_{22}, O\right\}
$$

it gives us the following Cayley table

\begin{tabular}{c|cccccc} 
& $I$ & $E_{11}$ & $E_{12}$ & $E_{21}$ & $E_{22}$ & $O$ \\
\hline$I$ & $I$ & $E_{11}$ & $E_{12}$ & $E_{21}$ & $E_{22}$ & $O$ \\
$E_{11}$ & $E_{11}$ & $E_{11}$ & $E_{12}$ & $O$ & $O$ & $O$ \\
$E_{12}$ & $E_{12}$ & $O$ & $O$ & $E_{11}$ & $E_{12}$ & $O$ \\
$E_{21}$ & $E_{21}$ & $E_{21}$ & $E_{22}$ & $O$ & $O$ & $O$ \\
$E_{22}$ & $E_{22}$ & $O$ & $O$ & $E_{21}$ & $E_{22}$ & $O$ \\
$O$ & $O$ & $O$ & $O$ & $O$ & $O$ & $O$
\end{tabular}

It follows immediately from the table that $T$ is a subsemigroup of $S$ which is a BZS inverse semigroup, with $E=\left\{I, E_{11}, E_{22}, O\right\}$ and $N=\left\{E_{12}, E_{21}, O\right\}$.

\section{Green's Relations}

Let us now obtain some basic properties on the Green's relations $\mathcal{R}, \mathcal{L}, \mathcal{H}, \mathcal{J}$ and $\mathcal{D}$ on a BZS semigroup $S$.

$$
R_{0}=L_{0}=H_{0}=D_{0}=J_{0}=\{0\}
$$

For any $x \in R_{0}$, we have that $x=x \cdot 1 \in x S^{1}=0 S^{1}=\{0\}$, which immediately implies that $R_{0}=\{0\}$. The other equalities follow similarly.

For $x, y \in E$ or $x, y \in N$

$x \mathcal{R} y \Rightarrow x y \mathcal{R} y x$

In fact, if $x, y \in E$, then, since $\mathcal{R}$ is a left congruence [1, Proposition 2.1.2],

and, if $x, y \in N$ then

$$
x \mathcal{R} y \Rightarrow\left\{\begin{array} { l } 
{ x x \mathcal { R } x y } \\
{ y x \mathcal { R } y y }
\end{array} \Rightarrow \left\{\begin{array}{l}
x \mathcal{R} x y \\
y x \mathcal{R} y
\end{array} \Rightarrow x y \mathcal{R} y x\right.\right.
$$

$$
x \mathcal{R} y \Rightarrow\left\{\begin{array} { l } 
{ x x \mathcal { R } x y } \\
{ y x \mathcal { R } y y }
\end{array} \Rightarrow \left\{\begin{array}{l}
0 \mathcal{R} x y \\
y x \mathcal{R} 0
\end{array} \Rightarrow_{(6)} \quad x y=0=y x \quad \Rightarrow \quad x y \mathcal{R} y x\right.\right.
$$

For $x, y \in E$ or $x, y \in N$

$$
x \mathcal{L} y \Rightarrow x y \mathcal{L} y x
$$

This follows similarly as in (7). 


\section{BOOLEAN ZERO SQUARE (BZS) SEMIGROUPS}

Note that properties (7) and (8) do not hold if one element is in $E$ and the other is in $N$. To see this, consider $N_{5}$ in Example 2, where the $\mathcal{R}$ classes of $N_{5}$ are $R_{u}=\{u, f\}, R_{e}=\{e, a\}$ and $R_{b}=\{b\}$, while its $\mathcal{L}$ classes are $L_{u}=\{u, e\}, L_{f}=\{f, a\}$ and $L_{b}=\{b\}$. From property (7) we have that $e \in E, a \in N, e \mathcal{R} a$ but $e a=a$ which is not $\mathcal{R}$ related with $a e=b$. Similarly, for property (8).

Theorem 1. Let $S$ be a BZS semigroup.

(1) If $a \in E$, then $R_{a} \cap E$ is a subsemigroup of $S$, which is a right zero semigroup. In particular, if $R_{a} \subseteq E$ then $R_{a}$ is a right zero semigroup.

(2) If $a \in N$, then $R_{a} \cup\{0\}$ is a subsemigroup of $S$.

Proof. (1): It is clear that $R_{a} \cap E$ is non-empty, since $a \in R_{a} \cap E$. For any $b, c \in R_{a} \cap E$, we have, $b \mathcal{R} a$ and $c \mathcal{R} a \Rightarrow b \mathcal{R} c \Rightarrow b=b b \mathcal{R} b c \Rightarrow b c \in R_{b}=R_{a}$

which means that, $R_{a}$ is a subsemigroup of $S$. Since $b$ and $c$ are idempotents, we have by [1, Proposition 2.3.3], that $b c=c$ and therefore $R_{a}$ is a right zero semigroup.

(2): It is clear that $R_{0}=\{0\}$ is a subsemigroup of $S$. So, it is enough to consider $a \in N \backslash\{0\}$, that is, $a \neq 0$ and $a^{2}=0$. For $b, c \in R_{a}$, there exist $x, y, z, w \in S^{1}$ such that

$$
a=b x, \quad b=a y, \quad c=a z \text { and } a=c w
$$

If, on one hand $b^{2}=0$, then

$$
a=b x \quad \Rightarrow \quad b a=b(b x)=b^{2} x=0 \cdot x=0 \quad \Rightarrow \quad b a=0
$$

and

$$
b c=b(a z)=(b a) z=0 \cdot z=0
$$

If, on the other hand $b^{2}=b$, then by [1, Proposition 2.3.3], $b c=c \in R_{a}$.

Therefore, $R_{a} \cup\{0\}$ is a subsemigroup of $S$.

Note that in general, an $\mathcal{R}$ class, $R_{x}$, is not a subsemigroup of $S$. In fact, if we consider the semigroup $N_{5}$ of Example 2, and its $\mathcal{R}$ class, $R_{e}=\{e, a\}$, where $e \in E$ and $a \notin E$, then we have that $a e=b \notin R_{e}$, which means that, $R_{e}$ is not a subsemigroup of $N_{5}$.

Theorem 2. Let $S$ be a BZS semigroup.

(1) If $a \in E$, then $L_{a} \cap E$ is a subsemigroup of $S$, which is a left zero semigroup. In particular, if $L_{a} \subseteq E$ then, $L_{a}$ is a right zero semigroup.

(2) If $a \in N$ then, $L_{a} \cup\{0\}$ is a subsemigroup of $S$.

Proof. Similar to the proof of Theorem 1.

Like in the note to Theorem 2, we can use Example 2 to illustrate that an $\mathcal{L}$ class of a BZS semigroup is not, in general, a subsemigroup of $S$.

Theorem 3. Let $S$ be a BZS semigroup.

(1) If $a \in E$, then $H_{a}$ is a group with only one element.

(2) If $a \in S \backslash E$, then $H_{a} \subseteq S \backslash E,\left(H_{a}\right)^{2}=\{0\}$ and $H_{a} \cup\{0\}$ is a subsemigroup of $S$.

(3) If a $\mathcal{D}$ class of $S$ contains an idempotent, all its $\mathcal{H}$ classes are singleton.

Proof. (1): In fact, by [1, Corollary 2.2.6], $H_{a}$ is a subgroup of $S$. We need to prove that $H_{a}$ has a unique element. For $a=0$ this is obvious, by (6). Let us now assume that $a \in E \backslash\{0\}$, and consider $b \in H_{a}$. We have that

If $b^{2}=0$ then

$$
b \mathcal{H} a \Rightarrow\left\{\begin{array} { l } 
{ b \mathcal { R } a } \\
{ b \mathcal { L } a }
\end{array} \Rightarrow \left\{\begin{array} { l } 
{ b b \mathcal { R } b a } \\
{ b a \mathcal { L } a a }
\end{array} \Rightarrow \left\{\begin{array}{c}
b^{2} \mathcal{R} b a \\
b a \mathcal{L} a
\end{array}\right.\right.\right.
$$

$$
b \mathcal{H} a \Rightarrow\left\{\begin{array} { l } 
{ 0 \mathcal { R } b a } \\
{ b a \mathcal { L } a }
\end{array} \Rightarrow \left\{\begin{array}{l}
b a=0 \\
a \in L_{b a}
\end{array} \Rightarrow a \in L_{b a}=L_{0}=\{0\} \Rightarrow a=0\right.\right.
$$

which is a contradiction. Therefore, we can conclude that $b^{2}=b$, and $b$ is an idempotent. Using again [1, Corollary 2.2.6], we conclude that $b=a$, and $H_{a}$ is a singleton subgroup of $S$.

(2): Let $a \in S \backslash E$, and consider $y \in H_{a}$, which cannot be equal to 0 , by (6). Then,

$$
y \mathcal{H} a \Rightarrow\left\{\begin{array} { l } 
{ y \mathcal { R } a } \\
{ y \mathcal { L } a }
\end{array} \Rightarrow \left\{\begin{array} { l } 
{ y y \mathcal { R } y a } \\
{ y a \mathcal { L } a a }
\end{array} \Rightarrow \left\{\begin{array}{c}
y^{2} \mathcal{R} y a \\
y a \mathcal{L} 0
\end{array} \Rightarrow y^{2} \in R_{0}=\{0\} \Rightarrow y \in N\right.\right.\right.
$$




\section{G.A. PINTO}

and therefore $H_{a} \subseteq S \backslash E$.

Again, with $a \in S \backslash E$ we have that

$$
b \mathcal{H} a \Rightarrow\left\{\begin{array} { l } 
{ b \mathcal { R } a } \\
{ b \mathcal { L } a }
\end{array} \Rightarrow \left\{\begin{array} { l } 
{ a b \mathcal { R } a a } \\
{ b a \mathcal { L } a a }
\end{array} \Rightarrow \left\{\begin{array} { l } 
{ a b \mathcal { R } 0 } \\
{ b a \mathcal { L } 0 }
\end{array} \Rightarrow \left\{\begin{array}{l}
a b=0 \\
b a=0
\end{array}\right.\right.\right.\right.
$$

Therefore, for $b, c \in H_{a}$, we can say that

$$
a b=0, \quad b a=0, \quad a c=0 \text { and } c a=0
$$

Since $b \mathcal{H} c$, we have that $b \mathcal{R} c$, and

$$
b \mathcal{R} c \mathcal{R} a \quad \Rightarrow \quad c b \mathcal{R} c c \mathcal{R} c a=0 \quad \Rightarrow \quad c b \mathcal{R} 0 \quad \Rightarrow \quad c b=0
$$

from which, we conclude that $\left(H_{a}\right)^{2}=\{0\}$ and that $H_{a} \cup\{0\}$ is a subsemigroup of $S$.

(3): This follows by [1, Lemma 2.2.3] and (1).

Note, that in Theorem 3(2) we verified that, for every $a \in N$, the $\mathcal{H}$ class $H_{a}$ is a subset of $N$. The same property does not hold for the $\mathcal{R}$ and $\mathcal{L}$ classes. In fact, the semigroup $N_{5}$ of Example 2 is such that $a \in N$, but $R_{a}=\{e, a\} \not \subset N$, as well as $L_{a}=\{f, a\} \not \subset N$.

Theorem 4. Let $S$ be a BZS semigroup.

(1) If $a \in S \backslash\{0\}$ and $D_{a} \subseteq E$, then $D_{a}$ is a subsemigroup of $S$.

(2) If $a \in S \backslash E$, then $D_{a} \cup\{0\}$ is a subsemigroup of $S$.

Proof. (1): For any $a \in S \backslash\{0\}$, we have by (6), that $D_{a} \neq\{0\}$. The fact that $D_{a} \subseteq E$ therefore implies that $D_{a} \subseteq E \backslash\{0\}$.

Considering any $b, c \in D_{a}$, there exist $d \in S$, such that $b \mathcal{L} d \mathcal{R} c$. By [1, Propositions 2.1.2 and 2.3.3], we have that

$$
d \mathcal{R} c \Rightarrow b d \mathcal{R} b c \Rightarrow b \mathcal{R} b c \Rightarrow b c \in R_{b} \subseteq D_{a}
$$

Thus, $D_{a}$ is a subsemigroup of $S$.

(2) Consider any $a \in S \backslash E$, that is, $a \neq 0$ and $a^{2}=0$. For any $b, c \in D_{a}$, there exist $d \in S$, such that $b \mathcal{L} d \mathcal{R} c$, which means, in particular, that $b=x d$ and $c=d y$ for some $x, y \in S^{1}$.

Also, $d \mathcal{R} c$ implies $b=x d \mathcal{R} x c$ and therefore $x c \in R_{b}$.

Then,

$$
b c=(x d)(d y)=x(d d) y
$$

If $d^{2}=d$, then $b c=x d^{2} y=x d y=x c \in R_{b} \subseteq D_{a}$.

If $d^{2}=0$, then $b c=0$.

Thus, $D_{a} \cup\{0\}$ is a subsemigroup of $S$.

\section{Special classes of BZS semigroups}

We now devote our attention to obtaining necessary and sufficient conditions for a BZS semigroup $S$ to be a band or an inverse semigroup. A characterisation of some BZS completely 0 -simple is presented. Also, the commutativity property will be approached.

Theorem 5. Let $S$ be a BZS semigroup. The following statements are equivalent:

(1) $S$ is a band;

(2) $(\forall x \in S) x^{3}=x$.

Proof. (1) $\Rightarrow(2)$ : The definition of a band tells us that $x^{2}=x$ for all $x \in S$. Then,

$$
x^{3}=x^{2} \cdot x=x \cdot x=x
$$

and the result follows.

$(2) \Rightarrow(1)$ : Take an element $x \in S$. Since $S$ is BZS, $x^{2}=x$ or $x^{2}=0$. If $x^{2}=x$, there is nothing to prove. If $x^{2}=0$, then $x=x^{3}=x^{2} x=0 \cdot x=0$ which, immediately implies that $x^{2}=x$, for every element of $S$, that is, $S$ is a band.

In the following Theorem and its proof, we use the identification provided from Rees Theorem [1, Theorem 3.2.3], for a completely 0 -simple semigroup $S$. Such $S$ is isomorphic to

$$
(I \times G \times \Lambda) \cup\{0\},
$$




\section{BOOLEAN ZERO SQUARE (BZS) SEMIGROUPS}

where $G$ is a group, $I$ and $\Lambda$ are non-empty index sets, and $P=\left[p_{\lambda i}\right]$ is a $\Lambda \times I$ sandwich matrix with entries in the zero group $G^{0}=G \cup\{0\}$. Also, every row and column of $P$ has at least a non-zero entry. The semigroup operation is defined by

$$
(i, a, \lambda)(j, b, \mu)=\left\{\begin{array}{ccc}
\left(i, a p_{\lambda j} b, \mu\right) & \text { if } & p_{\lambda j} \neq 0 \\
0 & \text { if } & p_{\lambda j}=0
\end{array}\right.
$$

Theorem 6. Let $S$ be a BZS semigroup. The following statements are equivalent:

(1) $S$ is a completely 0 -simple semigroup with no zero entries in the sandwich matrix;

(2) $S$ is a rectangular 0 -band.

Proof. (1) $\Longrightarrow(2)$ : Let $S$ be a completely 0-simple semigroup. Considering an arbitrary element $x$ in $G$, for any $\lambda \in \Lambda$ and $i \in I$, we have that $p_{\lambda i} \neq 0$. Then,

$$
(i, x, \lambda)^{2}=(i, x, \lambda)(i, x, \lambda)=\left(i, x p_{\lambda i} x, \lambda\right) \neq 0,
$$

which therefore implies, since $S$ is a BZS semigroup, that $(i, x, \lambda)^{2}=(i, x, \lambda)$. Thus,

$$
\begin{aligned}
(i, x, \lambda)(i, x, \lambda)=(i, x, \lambda) & \Leftrightarrow\left(i, x p_{\lambda i} x, \lambda\right)=(i, x, \lambda) \\
& \Leftrightarrow x p_{\lambda i} x=x \\
& \Leftrightarrow p_{\lambda i}=x^{-1}
\end{aligned}
$$

In particular, if we replace $x$ by the identity element of the group $1_{G}$, we obtain $p_{\lambda i}=1_{G}$, and therefore $x^{-1}=1_{G}$ which, is equivalent to $x=1_{G}$. So, $G$ is the trivial group.

Then, $S$ is isomorphic to $\left\{\left(i, 1_{G}, \lambda\right): i \in I\right.$ and $\left.\lambda \in \Lambda\right\} \cup\{0\}$, whose elements verify

$$
\left(i, 1_{G}, \lambda\right)\left(j, 1_{G}, \mu\right)=\left(i, 1_{G}, \mu\right) \quad \text { and } \quad\left(i, 1_{G}, \lambda\right) \cdot 0=0=0 \cdot\left(i, 1_{G}, \lambda\right)
$$

That is, $S$ is a rectangular 0-band.

$(2) \Rightarrow(1)$ : If $S$ is a rectangular 0-band, then

$$
(\forall a \in S)(\forall b \in S \backslash\{0\}) \quad a^{2}=a \quad \text { and } \quad a b a=a \quad .
$$

Then, for any $a, b \in S$ and $b \in S \backslash\{0\}$, we have that, $a=a b a \in S b S$ which implies that $S \subseteq S b S$.

Since, the reverse inclusion is always true, we can conclude that $S$ is a 0 -simple semigroup.

Also, if in $S \backslash\{0\}, a \leq_{n} b$, then $a b=b a=a$. We have that

$$
a b=b a \Rightarrow\left\{\begin{array} { l } 
{ a b a = b a a } \\
{ b a b = b b a }
\end{array} \Rightarrow \left\{\begin{array}{l}
a=b a \\
b=b a
\end{array} \Rightarrow a=b\right.\right.
$$

which, means that all non-zero idempotents are primitive, and therefore $S$ is completely 0 -simple.

Also, if $a, b \in S \backslash\{0\}$, then if $a b=0$ then $a b a=0 \neq a$, which is a contradiction. So, all the entries of the sandwich matrix are not zero.

It follows from the previous Theorem and its proof that for a BZS semigroup to be completely 0-simple where the sandwich matrix has no zero entries, it is necessary to have a singular group in the middle component of the Rees representation.

In fact, we can say that a BZS semigroup is completely 0 -simple where the sandwich matrix has no zero entries if, and only if, it is a completely simple semigroup with a zero adjoined.

Theorem 7. Let $S$ be a BZS semigroup. $S$ is an inverse semigroup if, and only if, the following conditions hold:

(1) $S$ is regular;

(2) $(\forall x \in S) x^{\prime} x^{2} x^{\prime}=x^{2}$, for any inverse $x^{\prime}$ of $x$.

Proof. Let $S$ be an inverse semigroup. Any element $x$ in $S$ has a unique inverse denoted by $x^{-1}$. By [1, Theorem 5.1.1], an inverse semigroup is a regular one, where the idempotents commute. So, by (2), $x^{2}$ is an idempotent that, therefore, commutes with $x x^{-1}$ and with $x^{-1} x$. Thus,

$$
\begin{aligned}
x^{4}=x^{2} & \Rightarrow x^{-1} x^{4}=x^{-1} x^{2} \Rightarrow x^{-1} x \cdot x^{2} \cdot x=x^{-1} x^{2} \Rightarrow x^{2} \cdot x^{-1} x \cdot x=x^{-1} x^{2} \\
& \Rightarrow x\left(x x^{-1} x\right) x=x^{-1} x^{2} \Rightarrow x^{3}=x^{-1} x^{2} \Rightarrow x^{3} x^{-1}=x^{-1} x^{2} x^{-1} \\
& \Rightarrow x^{2} \cdot x x^{-1}=x^{-1} x^{2} x^{-1} \Rightarrow x x^{-1} \cdot x^{2}=x^{-1} x^{2} x^{-1} \Rightarrow x^{2}=x^{-1} x^{2} x^{-1}
\end{aligned}
$$




\section{G.A. PINTO}

Conversely let us, assume that (1) and (2) hold. Let $e$ be an idempotent of $S$ and $e^{\prime}$ any inverse of $e$. By (2), we have that $e^{\prime} e^{2} e^{\prime}=e^{2}$, that is, $e^{\prime}=e$. Thus, we can conclude that each idempotent in $S$ has a unique inverse. Now, considering an element $x$ in $S$ and $x^{\prime}, x^{\prime \prime}$ inverses of $x$, we have that $x x^{\prime}$ and $x x^{\prime \prime}$ are idempotents and inverses of each other, as well as $x^{\prime} x$ and $x^{\prime \prime} x$. Thus, $x x^{\prime}=x x^{\prime \prime}$ and $x^{\prime} x=x^{\prime \prime} x$ and we can deduce that $x^{\prime}=x^{\prime}\left(x x^{\prime}\right)=x^{\prime}\left(x x^{\prime \prime}\right)=\left(x^{\prime} x\right) x^{\prime \prime}=\left(x^{\prime \prime} x\right) x^{\prime \prime}=x^{\prime \prime}$

The result follows, since by [1, Theorem 5.1.1], a regular semigroup where each element has a unique inverse is an inverse semigroup.

Theorem 8. Let $S$ be a BZS commutative semigroup. Then,

(1) $E$ is a subsemigroup of $S$;

(2) $N$ is an ideal of $S$;

(3) If $S$ is inverse then $x^{3}=x$, for every $x \in S$.

Proof. Consider any elements $x, y \in S$.

(1): If, on one hand, both belong to $E$, we have $x^{2}=x$ and $y^{2}=y$, and therefore

$$
(x y)^{2}=(x y)(x y)=x(y x) y=x(x y) y=(x x)(y y)=x^{2} y^{2}=x y
$$

which means that $x y \in E$, and therefore $E$ is a subsemigroup of $S$.

(2): If, on the other hand, for example $x \in N$, we have that

$$
(x y)^{2}=(x y)(x y)=x(y x) y=x(x y) y=(x x)(y y)=x^{2} \cdot y^{2}=0 \cdot y^{2}=0
$$

Thus, $x y \in N$ and we can conclude that $N$ is an ideal of $S$.

(3): If $S$ is an inverse commutative semigroup, any $x \in S$ has a unique inverse, $x^{-1}$, and we have by Theorem 7 (2), that

$$
x=x x^{-1} x x^{-1} x=x\left(x^{-1} x x x^{-1}\right)=x \cdot x^{2}=x^{3}
$$

We have seen previously that $x^{3}=x$ for all $x \in S$ holds in any BZS semigroup that it is also a band, or a commutative inverse semigroup. It also holds for a BZS completely 0-simple semigroup, where the sandwich matrix has no zero entries. However, this property does not hold for all the BZS semigroups. To see this, let us consider

$$
T=\left\{I, E_{11}, E_{12}, E_{21}, E_{22}, O\right\}
$$

of Example 4, which is an inverse BZS semigroup. Note that we have

$$
\begin{aligned}
& I^{3}=I, \quad E_{11}^{3}=E_{11}, \\
& E_{12}^{3}=O \neq E_{12}, \quad E_{21}^{3}=O \neq E_{21} \\
& E_{22}^{3}=E_{22} \text {, } \\
& O^{3}=O,
\end{aligned}
$$

from which, we can deduce that the mentioned property does not hold in all the BZS semigroups.

\section{Conclusion}

In this paper, we introduce a new class of ordered semigroups: BZS - Boolean Zero Square semigroups. Several basic properties on Green's relations are obtained. Necessary and sufficient conditions for a BZS semigroup to be a band and to be an inverse semigroup are obtained. A characterisation of a special type of BZS completely 0-simple semigroup is presented.

\section{Conflict of interest}

The author declares no conflict of interest.

\section{Acknowledgment}

I thank Sultan Qabbos University for providing facilities and to the referees for their comments that improved the qulity of this paper. 


\section{BOOLEAN ZERO SQUARE (BZS) SEMIGROUPS}

\section{References}

1. John, M. Howie, Fundamentals of Semigroup Theory, L.M.S. Monographs, 12, Oxford University Press, Oxford, 1995.

2. Farag, M. and Tucci, R. BZS Rings, Palestine Journal of Mathematics 2019, 8(2), 8-14.doi:10.7151/dmgaa.1243.

3. Farag, M. BZS Rings II, 2020 (preprint).

4. Bhavanari, S., Lungisile, G. and Dasari, N. Ideals and direct product of zero square rings, East Asian Mathematics Journal 24, 2008, 4, 377-387.

5. Blyth, T.S. and McFadden, R. Naturally ordered regular semigroups with a greatest idempotent, Proceedings of the Royal Society of Edinburgh, 91A, 1981, 107-122. doi:10.1017/S0308210500012671.

6. Blyth, T.S. Lattices and Ordered Algebraic Structures, (Springer 2005). doi:10.1007/b139095.

7. Clifford, A.H. and Preston, G.B. The Algebraic Theory of Semigroups, volume II, A.M.S. Mathematical Surveys, 1967. doi: 10.1090/surv/007.2.

Received 25 June 2020

Accepted 18 January 2021 